\title{
Splitless injection on capillary columns, Part II. Conditions and limits, practical realization
}

\author{
Grob, K ; Grob, G
}

\begin{abstract}
Our procedure of direct splitless injection on capillary columns is based on the choice of column temperature for injection. Under appropriate conditions a marked concentration effect is observed, i.e. the eluted band is more concentrated than the injected one. The dependence of this effect on column temperature, injection time, sample size, and other variables has been studied experimentally. A solvent bypassing technique has been developed which allows the performance of trace analysis on capillary columns
\end{abstract}

DOI: https://doi.org/10.1093/chromsci/7.10.587

Posted at the Zurich Open Repository and Archive, University of Zurich

ZORA URL: https://doi.org/10.5167/uzh-153883

Journal Article

Published Version

Originally published at:

Grob, K; Grob, G (1969). Splitless injection on capillary columns, Part II. Conditions and limits, practical realization. Journal of Chromatographic Science, 7(10):587-591.

DOI: https://doi.org/10.1093/chromsci/7.10.587 


\title{
Splitless Injection on Capillary Columns, Part II. Conditions and Limits, Practical Realization
}

\author{
by K. Grob and G. Grob, Department of Organic Chemistry, University of Zurich, Switzerland
}

\begin{abstract}
Our procedure of direct splitless injection on capillary columns is based on the choice of column temperature for injection. Under appropriate conditions a marked concentration effect is observed, i.e. the eluted band is more concentrated than the injected one. The dependence of this effect on column temperature, injection time, sample size, and other variables has been studied experimentally. A solvent bypass. ing technique has been developed which allows the performance of trace analysis on capiliary columns.
\end{abstract}

\section{Introduction}

Part I (1) of this study reported a simple method of sample injection without stream splitting. Conditions and limits of this method were not discussed in detail.

There is a strong belief in the rule that for analysis on capillary columns the liquid sample "should be introduced very sharply and evaporate instantaneously and completely" (2). Furthermore "a sharp sample plug," as produced by a properly designed stream splitter, should enter the column

The maximum volume $V_{i}$ of the vapor plug in the injection port with negligible effect on column performance is (3)

$$
V_{i},\left(V_{*} / \mathrm{n}\right) \cdot 0.6
$$

Under our experimental conditions $n-300,000, t_{1}-$ $15 \mathrm{~min}) \mathrm{V}_{\mathrm{i}}$ is approximately $0.1 \mathrm{ml}$. "The corresponding maximum injection time is of the order of 1 sec.

According to our experience this rule is correct for very short retention times and for isothermal analysis. For normal and longer retention. even under isothermal conditions, it is too severe. It no longer holds when during injection the column temperature is e.g. $50 \mathrm{C}^{\circ}$ below analysis temperature. Whether heating from injection temperature to analysis temperature is done rapidly or slowly is of no importance. This means that a much larger sample with proportionally lower concentration can be introduced into the column with. out specially designed trapping and without subsequent instantaneous vaporization

As far as we know there is no theoretical treatment of the problem available. According to current opinion the sample plug concentrated on the cooled column should broaden during warming, thus making the intermediate reduction of the column temperature ineffective. In this paper we describe in detail our observations as well as some application techniques de veloped.

\section{Apparatus and Materials}

Most of the tests were run on a glass capillary of $0.35 \mathrm{~mm}$, i.d., and $65 \mathrm{~m}$ length, coated with Emulphor
O (polyethylene glycol octadecylether). The separation number of this column for the pair tridecanetetradecane at $80^{\circ} \mathrm{C}$ and $6 \mathrm{ml} \mathrm{H}_{2}$ per min was 52 ( $\mathrm{n}=$ 260,000 ). The chromatographic equipment including stream splitter was the same described in Part I of this study.

The samples were members of the $n$-2-alkanones series. For gaseous sampling we prepared a mixture of $0.1 \mathrm{~g}$ butanone, $0.2 \mathrm{~g}$ 2-pentanone, etc. up to $3.2 \mathrm{~g}$ 2 -nonanone. The mixture was kept in a serum vial and gaseous samples were taken from the headspace with a common $10 \mu \mathrm{l}$ syringe. Solutions of equal amounts of alkanones in hexane were prepared for liquid sampling. The concentration of single compounds in the different solutions was $0.2 \%, 0.01 \%, 0.002 \%$ respectively.

\section{Experimental}

\section{Gaseous Samples, Influence of Injection Time}

We preferred gaseous samples to study the influence of sample injection time to the column to eliminate the problem of excess solvent. A constant amount of headspace gas, taken at room temperature, was injected either rapidly or over a measured time period. To assure equality between the manually adjusted injection time and the duration of sample introduction into the column, we used constant stream splitting for all injections. It must be emphasized that the object of this splitting was not the usual one but was to eliminate diffusion of the sample in the injection port with corresponding prolonged sample input into the column.

Figure 1A shows the chromatogram of a regular run with rapid injection and constant column temperature. Increasing the injection time up to 20 sec led to chromatogram 1B which shows broadened peaks, especially for the more volatile sample components. It is interesting to note, however, that the base width of the octanone peak is in the order of $10 \mathrm{sec}$, i.e. half the injection time. Thus more than a twofold concentration takes place for this component even under isothermal conditions.

This concentration effect is increased when the column temperature is lowered for injection. A temperature reduction of $50^{\circ}$ leads to a concentration sufficient for ideal resolution of those substances which

1. Grob, K. and Grob, G., J. Chromatog. Sci. 7, 584 (1969).

2. Ettre, L. S., "Open Tubular Columns in Gas Chromatography." Plenum Press, New York, 1965, p. 112.

3. Littlewood, A. B., "Gas Chromatography" Academic Press, New York and London, 1962, p. 164. 

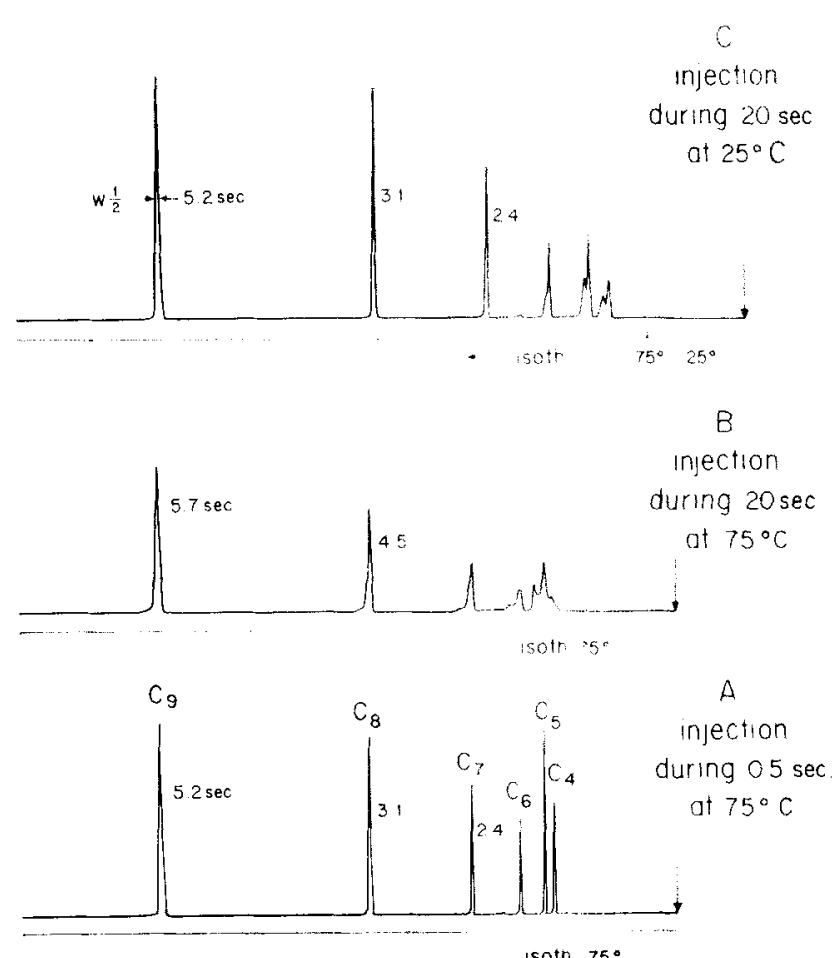

Figure 1. Column and chromatographic conditions see text. $2.0 \mu$ l of gaseous 2-alkanone mixture (butanone to nonanone; headspace) injected with constant split ratio 1:10. Column temperature for injection and injection time varied.

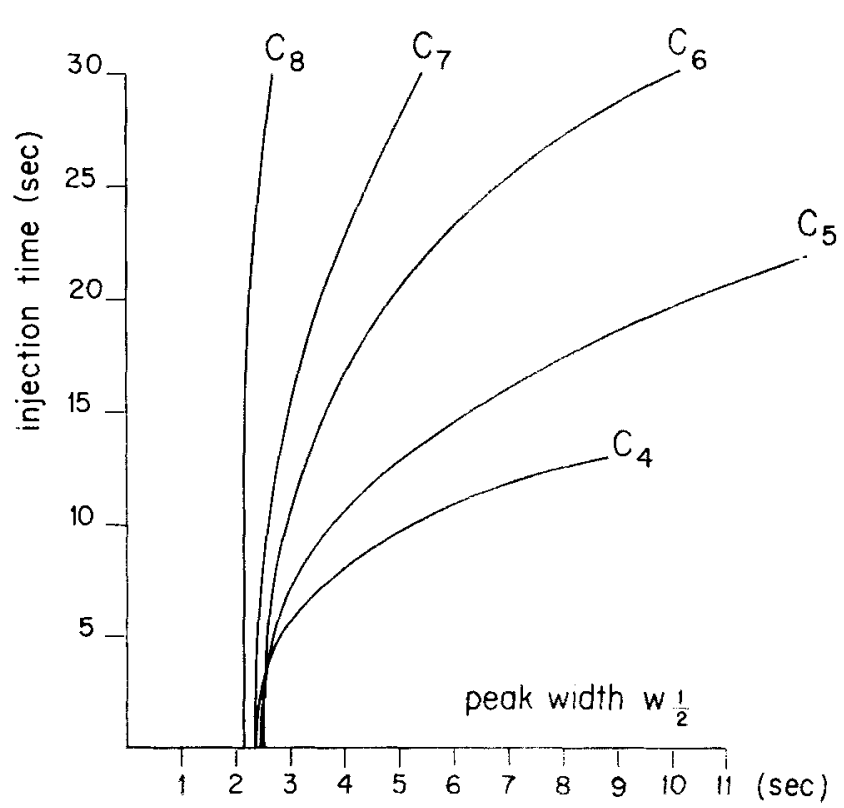

Figure 2. $2.0 \mu \mathrm{l}$ of gaseous alkanone mixture $\mathrm{C}_{4}-\mathrm{C}_{8}$. Column temperature during injection $25^{\circ}$, after injection temperature program $5^{\circ} / \mathrm{min}$. Effect of varied injection time on width of single peaks.

show reasonable retention at the given temperature of $75^{\circ}$ (i.e. heptanone and higher homologues). (Figure 1C.) More volatile samples are normally run at lower temperatures. These substances can also be well resolved after lowering the column temperature for slow injection. The increased peak heights in Figure $1 \mathrm{C}$ as compared to $1 \mathrm{~A}$ are due to decreased split ratio caused by decreased flow resistance at $25^{\circ} \mathrm{C}$.

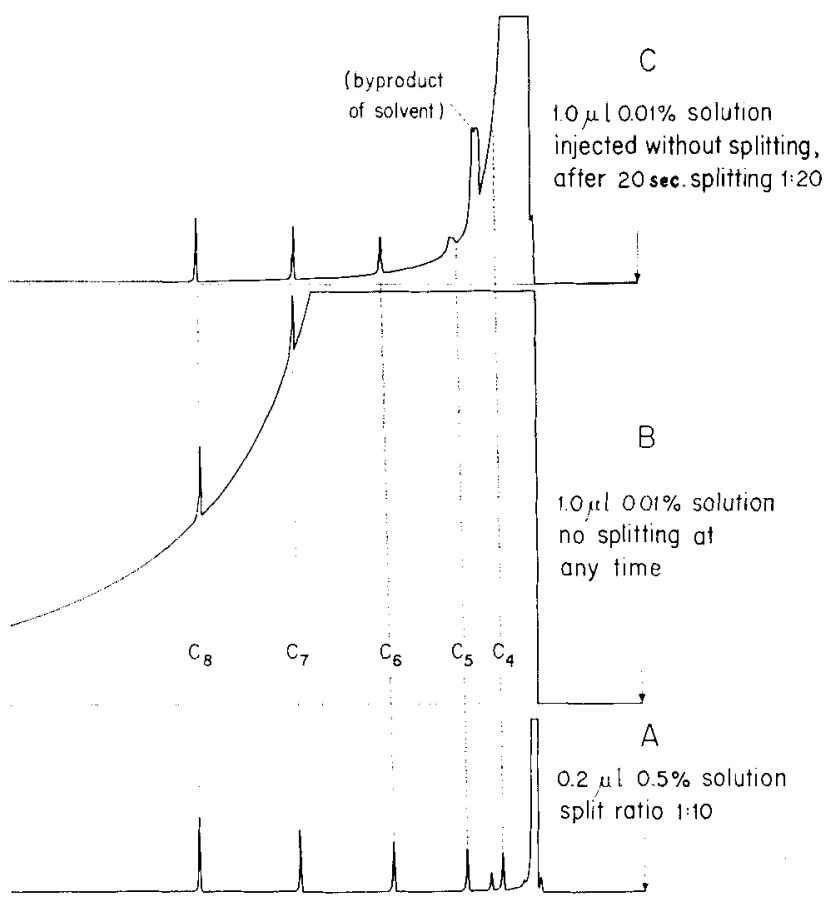

Figure 3. Liquid sample, alkanones $\mathrm{C}_{4}-\mathrm{C}_{8}$. Chromatographic conditions as for Figure 2. A: Regular procedure. B-C: Increased size of more dilute sample injected without splitting.

To improve the chromatographic conditions for the more volatile sample components, we used a temperature program of $5^{\circ}$ per min instead of heating rapidly from injection temperature to analysis temperature. For injection the column was held at $25^{\circ} \mathrm{C}$ as before, and the injection time was varied. From the chromatograms obtained this way we determined the relation between injection time and peak width for all components (Figure 2). Nonanone showed constant peak shape for all injection times used and was therefore left out of the sample. For butanone and pentanone, peak asymmetry grew to such an extent that for longer injection times the measurement of peak width became impossible. Figure 2 visualizes the concentration effect. Octanone, for example, after injection over $30 \mathrm{sec}$ at $25^{\circ} \mathrm{C}$, was eluted $20 \mathrm{~min}$ later at approximately $80^{\circ} \mathrm{C}$ with a base width of about $5 \mathrm{sec}$. This is practically identical with what is available from optimum performance of the column.

\section{Liquid samples, handling of excess solvent}

Figure 3 shows chromatograms obtained from liquid samples under conditions similar to those used for Figure 2, i.e. injection at $25^{\circ} \mathrm{C}$ followed by temperature programming at $5^{\circ} \mathrm{C}$ per min. Figure $3 \mathrm{~A}$ represents a normal run with a small amount of moderately dilute sample, injected with splitting. For splitless injection we used a solution 50 times more dilute. Direct injection of $1 \mu l$ produced Figure $3 B$. Note that the alkanone peaks are of perfect shape. Figure $3 \mathrm{C}$ was obtained in the same way as $3 \mathrm{~B}$ except that $20 \mathrm{sec}$ after injection the split valve was opened to clean the injection port. This procedure did not cause any detectable loss of alkanones. The apparent drastic difference between $3 \mathrm{~B}$ and $3 \mathrm{C}$ is obviously due only to the relatively small amount of solvent not introduced 
into the column after $20 \mathrm{sec}$ although a volume of carrier gas equal to twice the injector volume has been passed up to this time.

The first reasonably, though not perfectly, resolved peak is hexanone. In case of gaseous sampling and under identical flow and temperature conditions i Figure 2) the hexanone peak begins to broaden when the injection time is about $10 \mathrm{sec}$. During this time about $1 \mathrm{ml}$ of gas has been fed to the column. The gas volume (at column outlet) of the introduced liquid sample of $1 \mu \mathrm{l}$ hexane is $0.2 \mathrm{ml}$. Assuming a fivefold dilution with carrier gas, this would account for $10 \mathrm{sec}$ injection time. The assumption seems reasonable since, for our small column flow and large sample size, we used a relatively wide injector tube. We conclude froin this agreement that the concentration phenomenon is basically the same for gaseous as well as for liquid samples. Apparently it is not strongly affected by the large amount of solvent.

There is no doubt, on the other hand, that continuous heavy overloading will deteriorate the column. Since the sample components of interest after injection on a relatively cold column are concentrated in a first short length of the capillary, it seemed that it might be possible to vent the bulk of solvent by a bypass at the end of a relatively short inlet piece. As we recently reported (4) repeated cutting and reconnecting of capillaries can be done without any loss of performance. We therefore cut the column at $1.60 \mathrm{~m}$, measured from the inlet end, and reconnected it in the following manner: A $15 \mathrm{~mm}$ piece of Teflon shrink tubing (type WG 24) was fastened by warming on the end of the main column leaving $5 \mathrm{~mm}$ of unshrunk tubing beyond the end of the capillary. After putting in this open end a short piece of steel wire with the same diameter as the capillary, we warmed the remaining $5 \mathrm{~mm}$ of Teflon to produce a solid connection of glass and steel. It is easy to withdraw the wire provided its surface is smooth and the part of Teflon covering the wire is not too long. We then introduced the end of the inlet capillary into the shrunken Teflon and obtained a perfectly gas-tight connection that can be opened and reconnected many times by simply pulling out and reintroducing the end of the inlet capillary. We have prepared numerous connections of this kind which were used for about 30 operations and at oven temperature of $200^{\circ} \mathrm{C}$ without leaking. After 30 operations we renew the connection to avoid increasing distortion. The manipulation is facilitated by an appropriate shape of the parts connected see Figure 4 :

The procedure for solvent bypassing is as follows: the disconnected inlet part is fitted into the injection port. Its open end is joined to a flow restriction valve and a soap bubble meter by means of a thin plastic tube. In case of large solvent sizes we insert a glass tube filled with glass wool between capillary and flow restrictor to protect the restrictor from condensed solvent. By applying a low carrier gas pressure $1-0.1$ at) a constant flow, similar to normal column flow. is achieved with the split valve elosed. Injections of up to $1 \mu \mathrm{l}$ are made rapidly, Larger amounts are introduced over a few sec to avoid back-diffusion of the vapors. After an exactly measured time of, e.g., $30 \mathrm{sec}$ the carrier gas supply to the injection chamber is stopped. After another measured time. during which

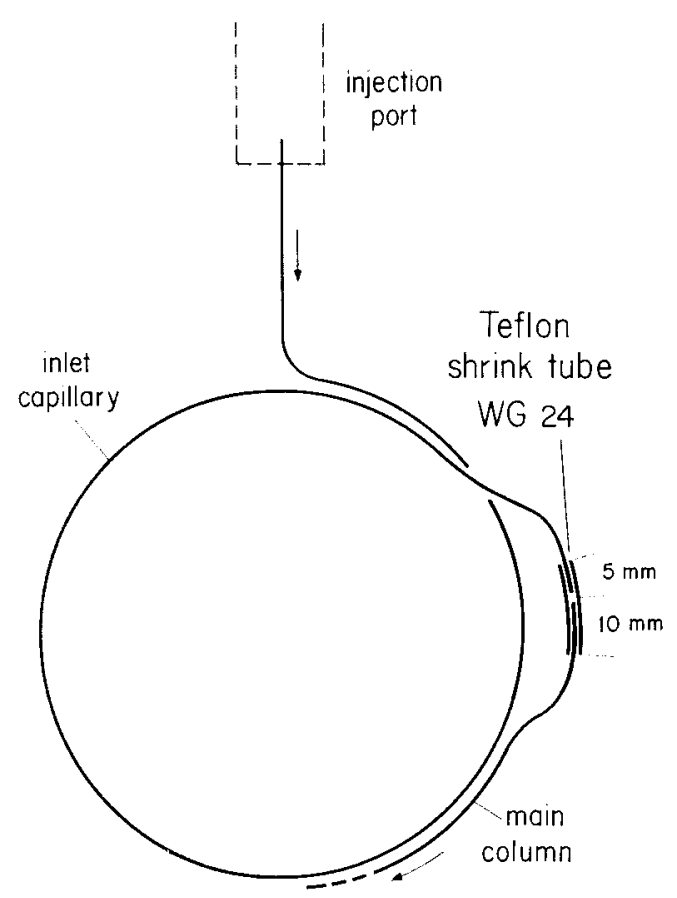

Figure 4. Schematic of connection between main column and short inlet capillary used for splitless injection with solvent bypassing.

the flow decreases to zero, the plastic tube is withdrawn, the inlet part is connected to the main column and normal flow conditions are resumed. The column is heated only after the air, which entered through the open end has been swept out of the cold column.

For a given inlet capillary (i.e. for a given length and diameter and film thickness) the important parameters in this procedure are:

a) the temperature of the inlet capillary,

b) the total gas flow through the inlet capillary,

c) the total sample size,

d) the relative volatility of the solvent as well as of the most volatile sample to be resolved.

The optimum parameters must be found experimentally. The critical point normally is the removal of the solvent from a sample containing very volatile components, as is the case with the example reported here. In this case either some loss of volatiles or a moderate removal of solvent must be accepted. In our case (Figure 5) the first two or three alkanones are tor volatile for prolonged injection (for liquid samples this means splitless) at room temperature. The injection parameters for the chromatograms in Figure 5 are, therefore, chosen to favour effective solvent removal, accepting approximately $80 \%$ loss of butanone and $60 \%$ loss of pentanone; the reduction of hexanone was less than $10 \%$. As for the chromatographic conditions, Figure 5A corresponds exactly to Figures 3B and $3 \mathrm{C}$ except for solvent bypassing. In contrast, the run $5 \mathrm{~B}$ accomplished with $5 \mu \mathrm{l}$ of $0.002 \%$ solution can in no way be duplicated without solvent bypassing.

4. Grob, K., and Grob, G., J. Chromatog. Sci., 7, 515 (1969) 


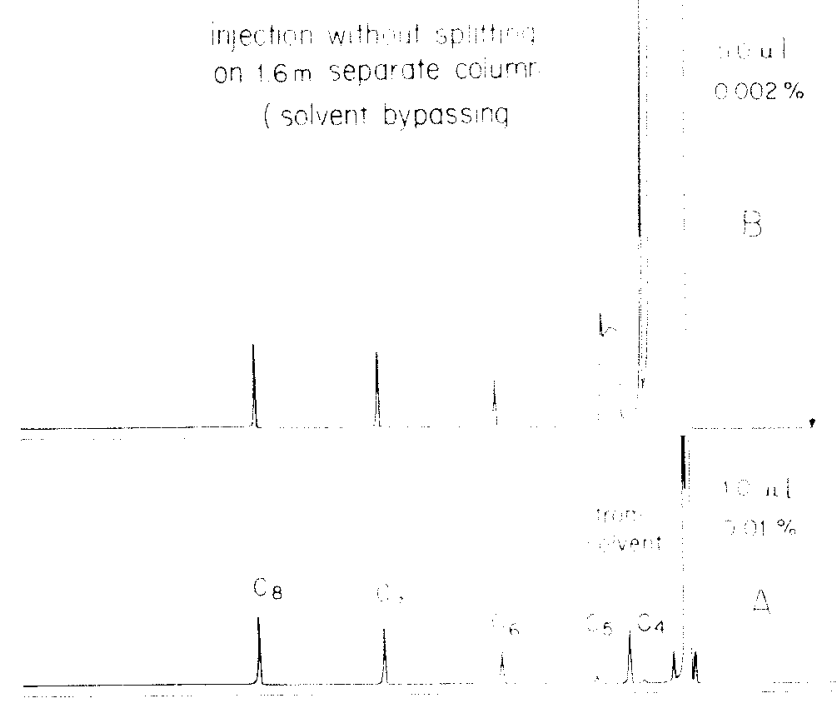

Figure 5. Liquid sample, alkanones $C_{4}-C_{3}$. Splitless injection with solvent bypassing. Size and dilution of sample varied.

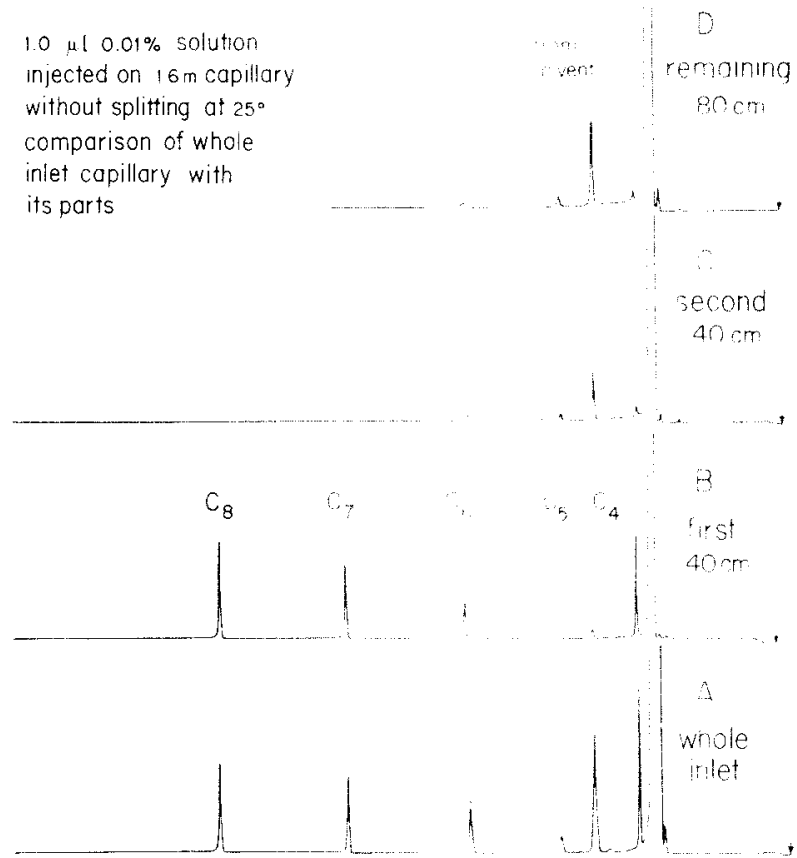

Figure 6. Sample and chromatographic conditions as for Figure 5A. A: Full solvent bypassing procedure. B-D: Single parts of loaded inlet capillary connected to column. For details of procedure see text.

Note that heptanone and octanone are perfectly resolved even under these drastic conditions, whereas hexanone $\mathrm{w}_{1 / 2}$ suffered an increase of $25 \%$.

We were interested in identifying the parts of the inlet capillary in which the single substances were concentrated. After running a chromatogram Figure $6 \mathrm{~A})$ from the whole $1.6 \mathrm{~m}$ inlet capillary, we prepared, under identical conditions, a second injection after which we cut the injection capillary into three parts of 40,40 , and $80 \mathrm{~cm}$. To prevent diffusion of the most volatile substances we first connected the last part $(80 \mathrm{~cm})$ to the column and ohtained figure $6 \mathrm{l}$ ). Wh handled the middle and the first part of the inlet in a corresponding fashion (Figures $6 \mathrm{C}$ and $6 \mathrm{~B}$ respectively?. The result was as follows: butanone had completely passed the first $40 \mathrm{~cm}$ and was found distributed over the remainder of the tube. More than half of it had been lost together with the solvent. Pentanone was detected over the entire tube. The same was observed for hexanone with marked accumulation in the first $40 \mathrm{~cm}$. Heptanone and octanone were exclusively present in the first quarter.

In summary, the result is not surprising since all well-resolved substances were concentrated on a relatively short piece of capillary. Normally it is sufficient, therefore, to use $50-100 \mathrm{~cm}$ inlet capillaries. For analysis of volatile substances, lowering the column temperature for injection is more effective than lengthening the inlet capillary.

\section{Practical recommendations for solvent bypassing}

To obtain the desired performance of the inlet capillary, carefully adjusted conditions are needed. It is inconvenient to reproduce these conditions (and primarily the exact and uniform temperature of the inlet piece) after every run. We prefer to carry out the injections on an independent apparatus where its conditions are continuously held at the optimum. This second apparatus need not be a gas chromatograph. Since most injections are performed at room temperature, only a heatable injection chamber with an efficient flow regulating device is needed. This arrangement also offers the opportunity of conveniently immersing the inlet capillary in a cooling bath for subambient injection temperature.

It is recommended that more than one inlet part for one column be prepared. During a run the next injection can be performed on a second inlet capillary.

In case of large sample sizes the coating of the inlet capillary may be damaged after a certain number of injections. This can easily be observed in glass capillaries (formation of minute droplets). Rinsing and recoating is done very rapidly since drying but no conditioning of the liquid phase is necessary. We renew the coating after about 20 injections of $5 \mu \mathrm{l}$ liquid samples with a polar solvent.

Except for very volatile substances there is little diffusion in the inlet capillary over a long time. We leave loaded inlet parts with stoppered ends at room temperature overnight without observing reduced resolution for substances with boiling points over $200^{\circ} \mathrm{C}$. We especially use this opportunity to carry loaded inlet parts over long distances to apparatus not equipped with an appropriate injection system for capillary columns ( in our case a combination of GC and MS). Another use of the same effect is taking head space samples at any location by simply drawing headspace gas through an inlet piece and carrying the latter to the laboratory for analysis.

Since efficient trace analysis is achieved with solvent bypassing, care is recommended to avoid unintentional analysis of silicone septum emollients. Even at injector temperatures of about $200^{\circ} \mathrm{C}$, most septa release sufficient bleed material to be concentrated on the capillary and to produce perfect and typical elution patterns. 


\section{Conclusions}

From our experimental results we deduce a concentration effect depending on the difference between column temperature and boiling point of sample. As far as we know, this effect has not yet been studied for its merits.

Besides boiling point, another essential parameter is functionality of the sample. When referring to boiling point only we presuppose that the sample is run on a column of very similar polarity.

In Part I we have expressed the rule that splitless injection is practicable if during injection the column temperature is at least $100^{\circ} \mathrm{C}$ below the boiling point of the most volatile sample component. This rule is confirmed by the observation that hexanone ib.p. $126^{\circ} \mathrm{C}$ ) is the most volatile alkanone reasonably resolved after splitless injection of a large amount of dilute solution on a column at room temperature see Figure $3 \mathrm{C}, 5 \mathrm{~A}, 5 \mathrm{~B}$ ). The rule holds for substances of any class provided the above-mentioned requirement is met.

Wishing to present average practical examples, we used a liquid phase and samples of medium polarity. Under nonpolar conditions, e.g. with alkanes on a squalane column, the concentration effect is even more pronounced.

Emulphor $\mathrm{O}$, the liquid phase used. is very viscous at $25^{\circ} \mathrm{C}$. This is the reason for the decreasing peak width with increasing $\mathrm{C}$-number measured after rapid injection (Figure 2). The concentration effect itself has no essential relation to viscosity (i.e. beyond the general chromatographic effects of viscosity). Hitherto we have coated the inlet capillaries with the same liquid phase and the same film thickness as the main column. We are unable to discuss the potential effects caused by changing these variables.

It is our experience that solvent bypassing seems to become more difficult with capillaries of smaller diameter. The influence of column diameter has not yet been studied extensively.

The advantages of our splitless injection procedure have been enumerated in Part I. We consider as the most important advantage the combination of the concentration effect with the solvent bypassing technique which broadens the application of capillary columns in trace analysis. The procedure does not offer a similar concentration power as that described by Novak, Vasak and Janak (5) but has the advantage of simplicity as well as of direct interpretation of the results.

\section{Acknowledgment}

We gratefully acknowledge the generous support of our work by F. J. Burrus \& Cie., Boncourt.

Manuscript received June 26, 1969.

5. Novak, J., Vasak, V. and Janak, J., Anal. Chem. 37, 660 (1965).

\title{
Liquid Ethylene-Propylene Copolymers as Stationary Phase in Gas Chromatography
}

\author{
by $\mathbf{O}$. Bieber, G. Degler, and H. Schnecko, Dunlop Forschungslaboratorium, Hanau, Germany
}

\section{Abstract}

The use of ethylene-propylene copolymers of lower molecular weight $([\eta] \sim 3)$ for the separation of hydrocarbon mixtures or pyrolysis products from polymers is reported. They can be used as stationary phase up to column temperatures of $350^{\circ} \mathrm{C}$.

\section{Introduction}

Oligomeric and polymeric material has found wide application in gas chromatography (1). Synthetic polymers (e.g. Teflon, polyethylene, cross-linked polystyrene) are used as solid support, and liquids (e.g. polyethylene glycols, silicones) have been employed as coatings.

Here, the application of a lower molecular weight random ethylene-propylene ( $\mathrm{EP}$ ) copolymer as liquid phase is reported. It can advantageously be used for the separation of aliphatic and aromatic hydrocarbons and of cracked polymer products up to column temperatures of $350^{\circ} \mathrm{C}$.

\section{Experimental}

The EP copolymers were prepared with a $\mathrm{VO} \mathrm{Cl}_{3} / \mathrm{Al}$ sesquichloride catalyst under atm $\mathrm{H}_{2}$. E-content was 52 mole- $\%,[\eta]=0.334$, corresponding to molwts of ca 15,000 . The columns were prepared by mixing the bulk copolymer liquid with Chromosorb (5 and $10 \%$ coating respectively). Gas chromatography was done on a Perkin-EImer F6 (isotherm, hot-wire detector, $250^{\circ} \mathrm{C}$ ) and on a Hewlett Packard F \& M 5750 instrument (temperature programmed, $2^{\circ} \mathrm{C} / \mathrm{min}, \mathrm{FID}$, $0.2-1.0 \mu \mathrm{l}$ injected); flash pyrolysis of polymers (ca 5 $\mathrm{mg}$ ) was conducted at $680^{\circ} \mathrm{C}$ for $18 \mathrm{sec}$.

\section{Results}

Figure 1 shows the separation power of the EPliquid phase packing for a normal test mixture of aro-

1. Leibnitz, E., and Struppe, H. G., "Handbuch der GasChromatographie," Verlag Chemie, Weinheim Bergstrasse, West Germany, 1967. 